\title{
Síndrome de Nicolau: a propósito de un caso
}

\section{Nicolau syndrome: a case report}

\author{
$M^{a}$ de la Paz Calot*, Antonela Dusso, Cecilia Ventrice y Paula Bourren \\ Unidad de Dermatología, Hospital de Agudos “Juan A. Fernández”, Buenos Aires, Argentina
}

\section{Resumen}

El síndrome de Nicolau es una complicación poco frecuente que surge tras la administración de diversos fármacos intramusculares, intraarticulares o subcutáneos. Se presenta el caso de un paciente de 30 años de edad, que luego de la aplicación de la tercera dosis de penicilina G benzatínica intramuscular desarrolló una placa equimótica con reticulado violáceo periférico en el sitio de inyección acompañado de efectos neurovasculares.

Palabras clave: Síndrome de Nicolau. Dermatitis livedoide. Penicilina G benzatínica.

\begin{abstract}
Nicolau syndrome is a rare complication caused by the intramuscular, intraarticular or subcutaneous administration of several drugs. We report the case of a 30-year-old patient, who after the intramuscular injection of the third dose of benzathine penicillin developed an equimotic plaque with violet peripheral reticulation at the aplication site accompanied by neurovascular effects.
\end{abstract}

Key words: Nicolau syndrome. Livedo-like dermatitis. Benzathine penicillin.

\section{Introducción}

El síndrome de Nicolau, también conocido como embolia cutis medicamentosa o dermatitis livedoide, es un síndrome infrecuente de origen yatrogénico. Aparece después de la aplicación de un fármaco por las vías intramuscular, intraarticular o subcutánea ${ }^{1-4}$. Inicia con eritema y dolor localizado en el sitio de inyección. Por lo regular evoluciona a una lesión livedoide que se torna hemorrágica, con necrosis de piel, grasa subcutánea y tejido muscular, que en ocasiones se acompaña de trastornos neurovasculares ${ }^{5}$.

\section{Presentación del caso}

Paciente de sexo masculino de 30 años de edad, con antecedentes de sífilis y consumo problemático de sustancias; consultó a la guardia del hospital de los autores por dolor intenso en la región glútea y miembro inferior izquierdo con impotencia funcional de éste, disuria y atonía del esfínter anal. La exploración física reveló eritema en glúteo, muslo y escroto a las 48 horas de la última aplicación de la inyección de penicilina $\mathrm{G}$ benzatínica intramuscular.
Correspondencia:

${ }^{*} \mathrm{M}^{\mathrm{a}}$ de la Paz Calot

E-mail: paz@calot.org

0210-5187/@ 2020 Colegio Ibero Latinoamericano de Dermatología A.C. (CILAD). Publicado por Permanyer. Este es un artículo open access bajo la licencia CC BY-NC-ND (http://creativecommons.org/licenses/by-nc-nd/4.0/).
Disponible en internet: 11-03-2021

www.MedicinaCutanealLA.com Med Cutan Iber Lat Am. 2020;48(3):169-172 
Con posterioridad evolucionó con la aparición de máculas y placas equimóticas, de bordes netos y contornos geográficos localizadas en glúteo y muslo, negativas a la vitropresión, que se extendieron rápidamente hasta comprometer el pie izquierdo (Fig. 1). Se confirmó tos seca y pérdida de peso de $5 \mathrm{~kg}$ en los últimos dos meses. Los estudios de laboratorio iniciales informaron leucocitosis, elevación de transaminasas, lactato deshidrogenasa y creatina-fosfocinasa y serología para VIH negativa.

En la ecografía de piel y partes blandas se reconoció un proceso inflamatorio del tejido celular subcutáneo sin colecciones ni masas definidas. La tomografía axial computarizada mostró líquido libre en zonas declives de la pelvis, engrosamiento mural a nivel rectal, hidrocele bilateral y, a nivel torácico, infiltrados micronodulillares bibasales con marcado derrame pleural izquierdo. Además, se solicitaron hemocultivos y muestra de esputo para bacilos resistentes al ácido-alcohol, la cual resultó positiva.

El paciente se internó en sala de clínica médica con diagnóstico de tuberculosis pulmonar y síndrome de Nicolau. Inició tratamiento antifímico, (isoniacida, rifampicina, pirazinamida y etambutol), diosmina, hesperidina (flavonoides micronizados) y antibióticos de amplio espectro (piperacilina/tazobactam, vancomicina y clindamicina) que se suspendió luego de obtener resultados negativos en los cultivos.

Evolucionó de forma favorable a los 15 días con recuperación de la función motora del miembro inferior izquierdo y control del esfínter anal (Fig. 2). Las lesiones avanzaron a la formación de costras hemorrágicas con posterior hiperpigmentacion residual.

\section{Discusión}

Freudenthal en 1924 y Nicolau en 1925 describieron este síndrome después de la inyección intramuscular de sales de bismuto para el tratamiento de un paciente con sífilis. Se han notificado múltiples casos relacionados con la administración intramuscular de diversos fármacos: antiinflamatorios no esteroideos, vitamina $\mathrm{B}$, glucocorticoides, vacunas, interferón recombinante, antibióticos, mesoterapia y escleroterapia ${ }^{6-8}$.

En el plano clínico cursa con dolor agudo intenso en el sitio de la inyección, seguido de la formación de una placa livedoide con márgenes bien delimitados. Por lo regular evoluciona a una placa reticulada y violácea con posterior necrosis cutánea en el sitio de punción, que se resuelve y deja una cicatriz atrófica ${ }^{9-11}$. Si bien

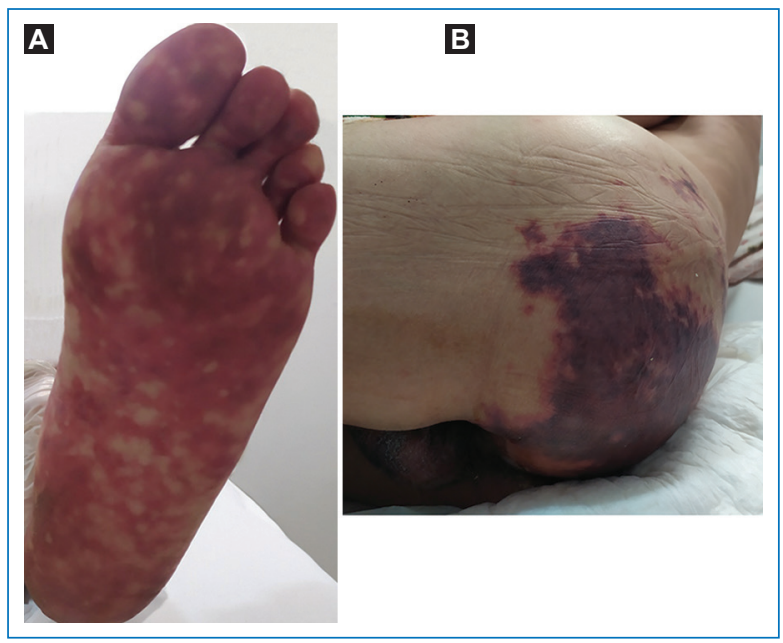

Figura 1. A-B: placa livedoide eritematoviolácea localizada en glúteo, planta y dorso del pie izquierdo.

la fisiopatología no está del todo dilucidada se han planteado diversas hipótesis:

- Estimulación nerviosa simpática secundaria al dolor causado por la inyección periarterial o intraarterial que causa vasoespasmo e isquemia.

- Las características farmacológicas de los antiinflamatorios no esteroideos inhiben la síntesis de prostaglandinas por medio de la ciclooxigenasa. La necrosis isquémica aparece luego de que estas sustancias inducen el vasoespasmo.

- Oclusión embólica secundaria a la inyección intraarterial.

- Inflamación perivascular por reacción citotóxica al fármaco.

- Obstrucción física inducida por compuestos lipofílicos que penetran los vasos sanguíneos de manera similar a la embolia grasa ${ }^{12}$.

La edad (niños menores de tres años) y la masa muscular disminuida son factores de riesgo predisponentes para desarrollar este síndrome ${ }^{13}$.

El diagnóstico es clínico. Pueden utilizarse métodos de imagen, como ultrasonido y resonancia magnética, con el fin de delimitar el área comprometida. En el estudio histopatológico se identifica necrosis por isquemia ${ }^{14,15}$.

La incidencia de las complicaciones luego de la inyección intramuscular varía entre 0.4 y $19.3 \%$. Son complicaciones frecuentes el sangrado en el sitio de aplicación, hematomas, dolor y formación de absce$\operatorname{sos}^{10}$. Dentro de los efectos neurovasculares se describen alteraciones de los reflejos osteotendinosos, atonía/hipotonía, alteraciones de la sensibilidad, 


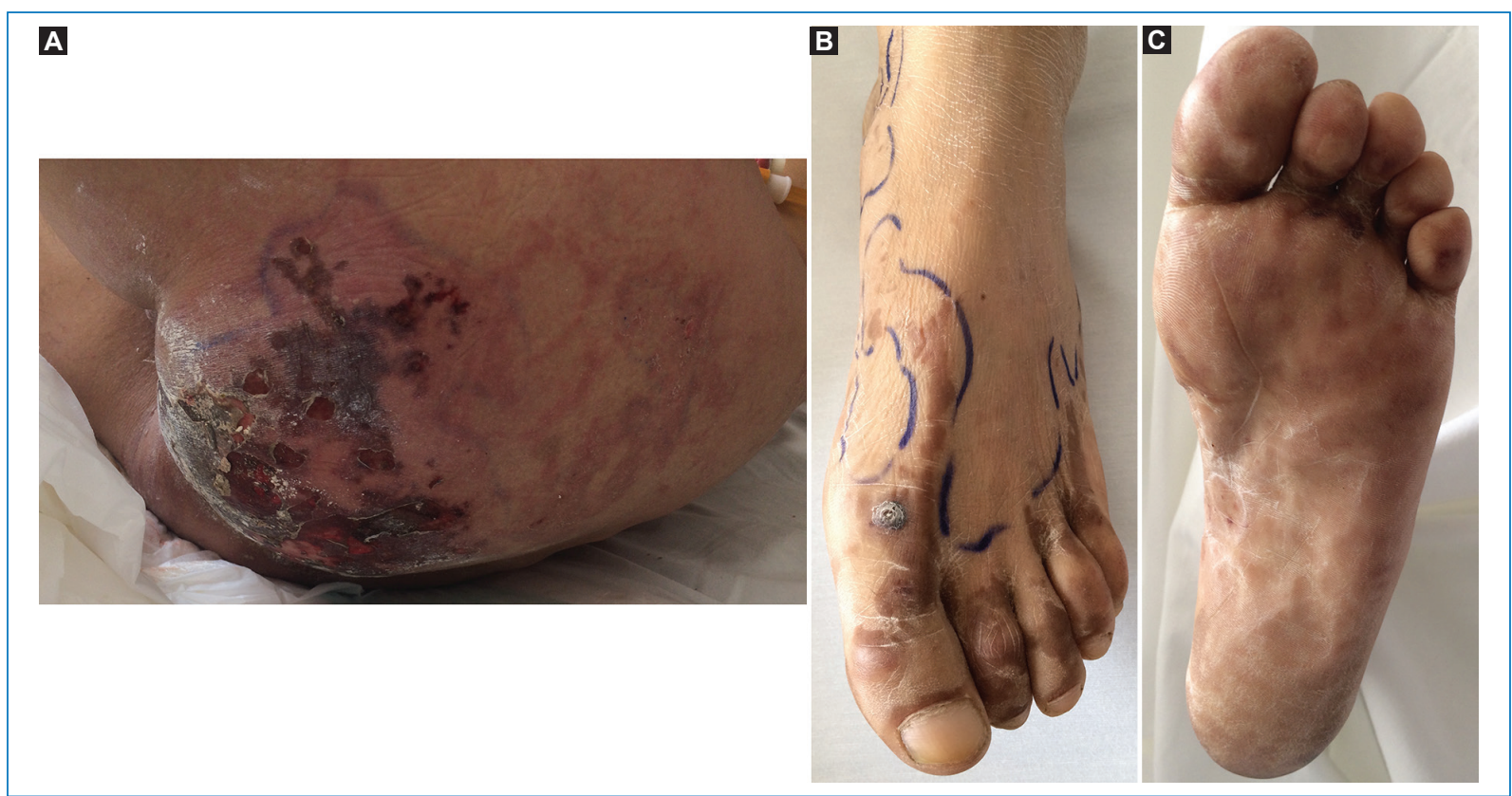

Figura 2. Evolución. A: costras y focos de necrosis cutánea en glúteo. B-C: reticulado livedoide residual en planta y dorso del pie izquierdo.

afectación esfintérica rectal o vesical, parálisis/plejía de las extremidades y caída del pie, entre otros. Con menor frecuencia, los pacientes pueden tener secuelas como dificultad para caminar e incontinencia rectal 0 urinaria $^{16}$.

El objetivo del tratamiento es disminuir la isquemia y limitar la necrosis cutánea. Si bien no existe un abordaje terapéutico regular, se utilizan fármacos como pentoxifilina, alprostadil, anticoagulantes (trombólisis con heparina), oxígeno hiperbárico y esteroides intralesionales ${ }^{13}$. Los antibióticos sistémicos se reservan en caso de sobreinfección bacteriana ${ }^{12}$. La aplicación de compresas frías puede agravar la necrosis por su capacidad de inducir vasoconstricción y producir isquemia ${ }^{17}$.

\section{Conclusiones}

Si bien no existen guías estandarizadas sobre el tratamiento y seguimiento de esta anomalía, es posible detectar y prevenir complicaciones si se establece un diagnóstico temprano y una atención oportuna. El personal de salud deber conocer las complicaciones que conlleva la aplicación de las inyecciones intramusculares. Es fundamental estar entrenado en la correcta técnica y evitar las indicaciones innecesarias, sobre todo en la población de riesgo.

\section{Conflicto de intereses}

Los autores confirman que no existe conflicto de intereses.

\section{Responsabilidades éticas}

Protección de personas y animales. Los autores declaran que para esta investigación no se han realizado experimentos en seres humanos ni en animales.

Confidencialidad de los datos. Los autores declaran que han seguido los protocolos de su centro de trabajo sobre la publicación de datos de pacientes.

Derecho a la privacidad y consentimiento informado. Los autores declaran que en este artículo se ha preservado la identidad de los pacientes pero no se ha logrado obtener el consentimiento informado.

\section{Bibliografía}

1. Cherasse A, Kahn MF, Mistrih R, Maillard H. Nicolau's syndrome after local glucocorticoid injection. Joint Bone Spine. 2003;70:390-392.

2. De Sousa R, Dang A, Rataboli PV. Nicolau syndrome following intramuscular benzathine penicillin. J Postgrand Med. 2008:54:332-334.

3. Hamilton B, Fowler P, Galloway H, Popovic N. Nicolau syndrome in an athlete following intra-muscular diclofenac injection. Acta Orthop Belg. 2008; $74: 860-864$.

4. Kresch-Tronik NS, De la Barreda F. Síndrome de Nicolau. Dermatol Rev Mex. 2012;56(5):332-334.

5. Gómez Alba V, Fernández J, Ferrán Pérez C, Santiago Rodríguez D, Mena F Síndrome de Nicolau. Manejo conservador. Caso clínico. Ciencia y Salud. [Internet]. 26 de julio de 2019 [citado 26 de julio de 2019];3(2): 57-63. Disponible en: https://revistas.intec.edu.do/index.php/cisa/article/view/1479 
6. Bellot B, Bonnet C, Retornaz K, Panuel M. Nicolau syndrome after intramuscular injection. Arch Pediatr. 2014;21(4):377-80.

7. Zaragoza J, Delaplace M, Benamara M, Estève E. A rare side effect of mesotherapy: Nicolau síndrome. Ann Dermatol Venereol. 2013;140(11) 713-7.

8. Stefanoa PC, Garellob M, Noltec MF, Lamyc P, Giglioc N, Castellanoc V, Gentile A. Síndrome de Nicolau por la administración de la vacuna séxtuple intramuscular en un lactante de 6 meses. Arch Argent Pediatr. 2017;115(1):e13-e16 / e13

9. Nischal KC, Basavaraj HB, Swaroop MR, Agrawal DP, Sathyanarayana BD Umashankar NP. Nicolau syndrome: an iatrogenic cutaneous necrosis. Journal of Cutaneous and Aesthetic Surgery. 2009;2(2):92-95.

10. Dadaci M, Altuntas Z, Ince B, Bilgen F, Tufekci O, Poyraz N. Nicolau syndrome after intramuscular injection of non-steroidal anti-inflammatorydrugs (NSAID). Bosn J Basic Med Sci. 2015;15(1):57-60.

11. Ingelmo J, Torrelo A, Zambrano A. Embolia cutis medicamentosa en un lactante por inmunización con DTP. Actas Dermosifiliogr. 2004;95(2):133-4

12. Kiliç I, Kaya F, Özdemir A. T, Demirel T, Celik I. Nicolau syndrome due to diclofenac sodium (Voltaren®) injection: a case report. Journal of Medical Case Reports. 2014;8, article 404
13. Discua-Guifarro D, Mejía-Irias C, Acosta Rosales J, López-Hernández C, Acosta-Zepeda R. Síndrome de Nicolau en paciente pediátrico posterior a la inyección intramuscular de penicilina benzatínica. Reporte de un caso. Archivos de Medicina 2015:11(4). [Acceso: 18 julio de 2016]. Disponible en: http://www.archivosdemedicina. com/medicina-de-familia/sindrome-de-nicolau-enpaciente-pediatricoposterior-a-la-inyeccin-intramusculardepenicilina-benzatinica-reporte-de-caso.pdf.

14. Gandino I, Majul B, Núñez J, Ravagna M, Muñoz S, Gianni M. Síndrome de Nicolau posterior a la inyección de penicilina intramuscular. Actualizaciones en SIDA. 2012;20(76):48-51.

15. Guarneri C, Polimeni G, Guarneri F, Cuzzocrea S. Embolia cutis medicamentosa following thiocolchicoside injection. Journal Compilation European Academy of Dermatology and Venereology. 2008;22: 1005-1006.

16. De Castro Miranda M, Rozenfeld S, Pacheco de Oliveira S. A systematic review of the non-allergic adverse reactions following benzathine benzyl penicillin injection. J Vasc $\mathrm{Br}$. 2004;3(3):253-60.

17. Şenel E, Ada S, Güleç AT, Ca冈lar B. Nicolau syndrome aggravated by cold application after i.m. diclofenac. Journal of Dermatology. 2008. 